2. - On the solvability of non-linear functional equations, Duke Math. J. (to appear).

3. - Variational boundary value problems for quasi-linear elliptic equations of arbitrary order, Proc. Nat. Acad. Sci. U.S.A. (to appear).

4. - Variational boundary value problems for quasi-linear elliptic equations. II, Proc. Nat. Acad. Sci. U.S.A. 50 (1963), 31-37.

5. - Variational boundary value problems for quasilinear elliptic equations. III, Proc. Nat. Acad. Sci. U.S.A. (to appear).

6. P. D. Lax and A. N. Milgram, Parabolic equations, Annals of Mathematics Study No. 33, Princeton, N. J., 1954, pp. 167-190.

7. G. J. Minty, Monotone (non-linear) operators in Hilbert space, Duke Math. J. 29 (1962), 341-346.

8. M. M. Vainberg and R. I. Kachurovski, On the variational theory of non-linear operators and equations, Dokl. Akad. Nauk 129 (1959), 1199-1202.

9. M. I. Visik, Solution of a system of quasilinear equations having divergence form under periodic boundary conditions, Dokl. Akad. Nauk 137 (1961), 502-505.

10. - Boundary value problems for quasilinear strongly elliptic systems in divergence form, Dokl. Akad. Nauk. 138 (1961), 518-521.

11. - Simultaneous quasilinear elliptic equations with lower order terms, Dokl. Akad. Nauk 144 (1962), 13-16.

12. M. I. Visik and O. A. Ladyzenskaya, Boundary problems for partial differential equations and some classes of operator equations, Uspehi Mat. Nauk 111 (1956), 41-97.

INSTITUTE For ADVANCEd STUdy AND UNIVERSITY OF CHICAGo

\title{
ERRATUM, VOLUME 48
}

Arnold N. Lowan, Herbert E. Salzer and Abraham Hillman, $A$ table of coefficients for numerical differentiation, pp. 920-924.

$$
\text { Page } 924, A_{m, 8} \text { for } m=18, s=20 \text {, reads } \frac{117}{4} \text {, should read } \frac{177}{4} \text {. }
$$

